\title{
Microcutting Redox Profile and Anatomy in Eucalyptus spp. With Distinct Adventitious Rooting Competence
}

\author{
Johnatan Vilasboa ${ }^{1 \dagger}$, Cibele Tesser da Costa ${ }^{1 \dagger}$, Leonardo Girardi Ransan ${ }^{1}$, \\ Jorge Ernesto de Araújo Mariath ${ }^{2}$ and Arthur Germano Fett-Neto ${ }^{1 *}$
}

OPEN ACCESS

Edited by:

Hélia Cardoso,

University of Evora, Portugal

Reviewed by:

Weibiao Liao,

Gansu Agricultural University, China

Ada Ricci,

University of Parma, Italy

*Correspondence:

Arthur Germano Fett-Neto

fett.neto@ufrgs.br

tThese authors have contributed equally to this work

Specialty section:

This article was submitted to Plant Development and EvoDevo,

a section of the journal

Frontiers in Plant Science

Received: 23 October 2020 Accepted: 28 December 2020

Published: 20 January 2021

Citation:

Vilasboa J, da Costa CT, Ransan LG, Mariath JEA and Fett-Neto AG (2021)

Microcutting Redox Profile and

Anatomy in Eucalyptus spp. With

Distinct Adventitious Rooting

Competence.

Front. Plant Sci. 11:620832.

doi: 10.3389/fp/s.2020.620832
${ }^{1}$ Plant Physiology Laboratory, Center for Biotechnology and Institute of Biosciences (Department of Botany), Federal University of Rio Grande do Sul, Porto Alegre, Brazil, ${ }^{2}$ Plant Anatomy Laboratory, Institute of Biosciences (Department of Botany), Federal University of Rio Grande do Sul, Porto Alegre, Brazil

Adventitious root (AR) development takes place in an intricate cellular environment. Reactive oxygen species (ROS) and antioxidant defenses, triggered by wounding in cuttings, can modulate this process. A comparative assessment of biochemical and anatomical parameters at critical rooting stages in hard- (Eucalyptus globulus Labill.) and easy(Eucalyptus grandis W. Hill ex Maiden) to-root species was carried out. Microcuttings from seedlings were inoculated in auxin-free AR induction medium and, after $96 \mathrm{~h}$, transferred to AR formation medium for a period of $24 \mathrm{~h}$. Samples were collected upon excision (Texc) and at the 5th day post excision (Tform). Delayed xylem development, with less lignification, was recorded in E. globulus, when compared to E. grandis, suggesting lower activity of the cambium layer, an important site for AR development. Superoxide was more densely present around the vascular cylinder at both sampled times, and in greater quantity in E. globulus than E. grandis, declining with time in the former. Hydrogen peroxide was localized primarily along cell walls, more intensely in the primary xylem and phloem, and increased significantly at Tform in E. globulus. Ascorbate peroxidase (APX), superoxide dismutase (SOD), and catalase (CAT) activities were generally higher in E. grandis and varied as a function of time in E. globulus. Soluble guaiacol peroxidase (GPRX) activity increased from Texc to Tform in both species, whereas cell wall-bound GPRX activity increased with time in E. grandis, surpassing E. globulus. Flavonoid content increased with time in E. grandis and was higher than E. globulus at Tform. Principal component analysis showed that species- and time-derived differences contributed to almost $80 \%$ of the variance. Overall, data indicate that $E$. grandis shows higher cambium activity and tighter modulation of redox conditions than E. globulus. These features may influence ROS-based signaling and phytohormone homeostasis of cuttings, thereby impacting on AR development. Besides being players in the realm of AR developmental differences, the specific features herein identified could become potential tools for early clone selection and AR modulation aiming at improved clonal propagation of this forest crop.

Keywords: rooting, Eucalyptus, reactive oxygen species, antioxidant defense, wounding, xylem, flavonoid 


\section{INTRODUCTION}

Adventitious roots (ARs) are formed post-embryonically from stems, leaves, hypocotyls, and other organs or tissues where previously there were no roots (Bellini et al., 2014; Steffens and Rasmussen, 2016). AR development is a complex process that involves several phytohormones, taking part in different steps of AR formation (Da Costa et al., 2013). Auxins, however, have proven to be central players acting synergistically or antagonistically with other phytohormones to stimulate AR initiation and development (Pacurar et al., 2014). Two main steps are recognized in AR formation. The first is known as the induction phase, in which high auxin levels are required and biochemical changes take place. This stage is followed by the formation phase, when cell division and differentiation occur, leading to AR primordium development and, finally, AR emergence (Da Costa et al., 2013). AR formation occurs naturally in some species, mainly in monocots, in which the primary root system is important for seedling development, but is shortly replaced by ARs, forming a fibrous root system (Bellini et al., 2014). Still, various conditions can induce it, such as flooding, etiolation, burial, soil chemicals, nutrient deficiency, or wounding (Steffens and Rasmussen, 2016).

The physical damage caused by wounding triggers several biochemical and physiological signals that lead to a systemic response. Reactive oxygen species (ROS), initially perceived only as toxic, have been shown to act as important signal transduction molecules in wound response (Mittler et al., 2011). The balance between toxic, non-toxic, or signaling depends on the action of ROS-scavenging mechanisms. These modulators can detoxify excessive ROS derived from cellular metabolism or ROS-producing enzymes (Mittler et al., 2004). Enzymatic antioxidants such as superoxide dismutase (SOD), catalase (CAT), ascorbate peroxidase (APX), and guaiacol peroxidase (GPRX) play important roles in ROS-scavenging capacity. SOD helps maintain the redox balance by the dismutation of superoxide radical $\left(\mathrm{O}_{2}{ }^{--}\right)$into hydrogen peroxide $\left(\mathrm{H}_{2} \mathrm{O}_{2}\right)$ and molecular oxygen $\left(\mathrm{O}_{2}\right.$; Kapoor et al., 2019). APX and CAT are fundamental to detoxify and maintain adequate cellular levels of $\mathrm{H}_{2} \mathrm{O}_{2}$ for normal plant growth and development (Anjum et al., 2016). APX uses ascorbic acid as an electron donor to detoxify $\mathrm{H}_{2} \mathrm{O}_{2}$, whereas CAT can directly degrade it. GPRX uses several substrates as reducers to eliminate $\mathrm{H}_{2} \mathrm{O}_{2}$ but can also produce ROS (Gechev et al., 2006). Flavonoids are a group of plant phenolics with non-enzymatic antioxidant properties, which can inactivate ROS generated during abiotic stress (Vicente and Boscaiu, 2018).

Participation of ROS in adventitious rooting is still poorly understood. Some lines of evidence indicate that $\mathrm{H}_{2} \mathrm{O}_{2}$ might act as a signaling molecule, inducing AR formation (Li et al., 2009; Huang et al., 2020). In this process, auxin is involved and thought to modulate AR growth through ROS accumulation, which is counterbalanced by a mutual regulation between ROS, nitric oxide (NO), and auxin to maintain cell redox homeostasis and signaling (Correa-Aragunde et al., 2016).

Wound-induced roots are especially important for plant propagation of economically relevant species in forest and horticulture industries (Steffens and Rasmussen, 2016; Vilasboa et al., 2018). This is the case of eucalyptus propagation, which is mainly vegetative and widely employed in the commercial forest industry for uniform cutting production from select superior genotypes. Eucalypt trees serve as one of the major sources of wood for timber, pulp, cellulose, and paper industries worldwide (Vilasboa et al., 2018). Eucalypt wood has also emerged as feedstock for chemicals, fuels, and other materials through biorefinery technologies (Penín et al., 2020). Out of ca. 900 known Eucalyptus species, Eucalyptus globulus stands out for its high pulp yield, wood density, and fiber quality (Carrillo et al., 2018). Low lignin levels and high syringyl/ guaiacyl $(\mathrm{S} / \mathrm{G})$ residue ratio contribute to the high pulp yield (Rencoret et al., 2007). However, vegetative propagation of this species and some of its hybrids is often limited due to its relatively low ability to produce ARs (De Almeida et al., 2015).

Although it is known that ROS can play important roles in AR development, this oxidative component has not been examined in detail in Eucalyptus cuttings. In this work, an in vitro system was used to perform a morphological and biochemical comparison of the easy-to-root Eucalyptus grandis and hardto-root E. globulus species in an attempt to better understand the dynamics of wound induced ROS and AR formation. The hypotheses tested were: (i) Microcutting anatomy features are related to root regeneration capacity; (ii) particular changes in ROS-related parameters of cuttings occur between the time of severance and early AR development; and (iii) distinct profiles of ROS-related parameters are apparent in eucalypt species of contrasting root regeneration capacity. The range of assessed parameters revealed specific patterns associated with each species and stage of AR development and may provide tools to characterize and possibly modulate rooting recalcitrance.

\section{MATERIALS AND METHODS}

\section{Plant Material}

Eucalyptus globulus Labill. and E. grandis W.Hill ex Maiden were used in the experiments. Seed surface sterilization and germination procedures were carried out according to De Almeida et al. (2015), with minor modifications. Surface-sterilized seeds were placed in groups of 15 distributed in approximately 30 to 40 glass jars of $300 \mathrm{ml}$ containing $60 \mathrm{ml}$ of half-strength MS salts (Murashige and Skoog, 1962) at pH 5.8 and $0.7 \%$ agar. Before sowing, jars were capped with a double layer of aluminum foil, autoclaved at $121^{\circ} \mathrm{C}$ for $20 \mathrm{~min}$. After sowing, jars were sealed with plastic film at the limit of foil and glass surface. Seedlings were kept under $16 \mathrm{~h}$ photoperiod of $100 \mu \mathrm{mol} \mathrm{m}{ }^{-2} \mathrm{~s}^{-1}$ photosynthetically active radiation (provided by white LED lights) and temperature of $23 \pm 2^{\circ} \mathrm{C}$.

\section{Adventitious Rooting}

For in vitro adventitious rooting, $3-\mathrm{cm}$ apical microcuttings with only two fully expanded leaf pairs were obtained by excision with a scalpel blade (Supplementary Figure 1). Remaining leaves were excised. Since the two species grow at different rates, E. globulus was excised with 14 weeks and 
E. grandis with 16 weeks after sowing, unless otherwise stated. The rooting culture system comprised two sequential steps, essentially as previously described (Fett-Neto et al., 2001; De Almeida et al., 2015) with modifications. First, cuttings were kept in induction medium containing $0.3 \mathrm{x}$ MS salts, $40 \mathrm{mg} \mathrm{l}^{-1}$ thiamine- $\mathrm{HCl}, 100 \mathrm{mg} \mathrm{l}^{-1}$ myo-inositol, and $0.6 \%$ agar for $96 \mathrm{~h}$. Then, cuttings were transferred to formation medium, identical in composition but with the addition of $0.1 \%$ activated charcoal. Media were devoid of sucrose and auxin, and $\mathrm{pH}$ was adjusted to 5.8 before autoclaving at $121^{\circ} \mathrm{C}$ for $20 \mathrm{~min}$. Adventitious rooting experiments were carried out in $20 \mathrm{ml}$ glass vials containing $6 \mathrm{ml}$ of medium and one cutting. Vials were sealed and kept under controlled conditions as mentioned in Plant Material section.

\section{Sampling}

Microcuttings were harvested at the moment of excision $(0 \mathrm{~h}$ induction stage, henceforth Texc) and $24 \mathrm{~h}$ after the transfer to formation medium ( $24 \mathrm{~h}$ into the formation stage, henceforth Tform). The latter harvest point was chosen as it generally coincides with early AR primordia development (De Almeida et al., 2015). For biochemical analyses, samples were weighed, immediately frozen in liquid nitrogen and stored at $-80^{\circ} \mathrm{C}$. Each biological replicate was composed of five whole microcuttings and five replicates were used. For morphological analyses, plants were kept in formation medium for 20 days. Three independent experiments were performed $(n=20)$ to quantify rooting percentage, length of the longest root, and root number. For anatomical analysis and lignin staining, microcutting stem bases were fixed under vacuum in McDowell and Trump's fixative solution of $4 \%$ formaldehyde and $1 \%$ glutaraldehyde in sodium phosphate buffer, pH 7.2 (McDowell and Trump, 1976), and kept at $4^{\circ} \mathrm{C}$. For ROS staining, microcutting stem bases were immediately used for histological sectioning in a hand microtome (Ranvier type) with the help of a soft wedge to hold samples in place. Twelve replicates were used for anatomical evaluations with lignin staining and three for ROS localization.

\section{Lignin Staining}

Cross sections of $10-15 \mu \mathrm{m}$ were obtained from fixed material using a handheld microtome. Histological sections were kept in distilled water to avoid dehydration and then transferred to spot plates for staining with phloroglucinol and $\mathrm{HCl}$, according to Johansen (1940), with minor modifications. A few drops of $1 \%$ phloroglucinol in $95 \%$ ethanol $[\mathrm{v} / \mathrm{v}]$ were applied to the sections, followed by a few drops of $30 \% \mathrm{HCl}$. After $5 \mathrm{~min}$, slides were prepared in water and images were immediately captured. The histological sections were observed using a Leica DMR microscope, coupled with digital image capture system DFC500 (Leica), using 20x or 40x long working distance objective lenses.

\section{ROS Localization}

Protocols for ROS visualization were adapted from Romero-Puertas et al. (2004). Histological cross sections were obtained as mentioned in Lignin staining section from fresh microcutting bases. Immediately, sections were transferred from distilled water to $1 \mathrm{mM}$ ascorbic acid in spot plates for $10 \mathrm{~min}$. Then, for superoxide staining, sections were immersed for $1 \mathrm{~h}$ in a solution of $1.41 \mathrm{mg} \mathrm{ml}^{-1}$ nitro blue tetrazolium (NBT), after which dark blue spots indicated NBT reduction. For $\mathrm{H}_{2} \mathrm{O}_{2}$ staining, separate sections were immersed in $1 \mathrm{mg} \mathrm{ml}$ 3,3'-diaminobenzidine (DAB) in $50 \mathrm{mM}$ phosphate buffer $(\mathrm{pH}$ 7.0) for $30 \mathrm{~min}$ under LED lighting. Oxidized DAB produces a distinct brownish precipitate. For chlorophyll removal, sections were then transferred to $95 \%$ ethanol $[\mathrm{v} / \mathrm{v}]$ for $15 \mathrm{~min}$. Slides were prepared in water and images were obtained as described in Lignin staining section.

\section{Image Processing and Area Measurements}

All area measurements of images obtained through photomicrography were done using Image $2 \mathrm{v} 1.53 \mathrm{c}$ (Rueden et al., 2017) and distribution Fiji (Schindelin et al., 2012). Xylem area percentage $(n=12)$ was defined as the area ratio of xylem tissue and the whole section. NBT- and DAB-stained area percentages were estimated using the Color Threshold feature of ImageJ2.

\section{Enzyme Activity Assays}

Total protein extract was obtained from approximately $100 \mathrm{mg}$ of frozen plant tissue. Samples $(n=5)$ were ground in liquid nitrogen and solubilized in $1.5 \mathrm{ml}$ extraction buffer [50 mM HEPES - pH 7.4, 1\% polyvinylpyrrolidone, $1 \mathrm{mM}$ EDTA, and 0.1\% Protease Inhibitor Cocktail (Sigma, United States)]. After centrifugation at $12,800 \times g$ at $4^{\circ} \mathrm{C}$ for $15 \mathrm{~min}$, the supernatant was recovered and immediately used for enzymatic activity assays. Protein content was determined according to Bradford (1976). Spectrophotometric readings were obtained with Spectramax M2 Microplate Reader (Molecular Devices, United States).

Ascorbate peroxidase activity was assayed according to Nakano and Asada (1981). Reaction mixture contained $2 \mathrm{ml}$ of buffer (50 mM phosphate buffer - pH 7.0, $0.5 \mathrm{mM}$ ascorbic acid, and $1 \mathrm{mM} \mathrm{H}_{2} \mathrm{O}_{2}$ ) and $50 \mu \mathrm{l}$ of the total protein extract ( $\sim 56 \mu$ g protein). Changes in absorbance due to ascorbic acid oxidation (using $\varepsilon=2,800 \mathrm{M}^{-1} \mathrm{~cm}^{-1}$ ) were monitored at $290 \mathrm{~nm}$.

Two fractions of guaiacol peroxidase were assayed, following different extraction protocols. Cell wall-bound GPRX activity was measured using an extract enriched for cell wall-bound protein, as described in Dos Santos et al. (2004). Briefly, pellets obtained after supernatant recovery were profusely washed until no soluble GPRX activity could be detected. Pellets were then incubated in $1 \mathrm{ml} 1 \mathrm{M} \mathrm{NaCl}$ at $4^{\circ} \mathrm{C}$ for $1 \mathrm{~h}$. The supernatant recovered after 15 min centrifugation at $10,000 \times g$ was used for activity determination as described by Cakmak and Marschner (1992). To $1.5 \mathrm{ml}$ of reaction buffer (50 $\mathrm{mM}$ phosphate buffer $\mathrm{pH} 7.0,0.05 \%$ guaiacol, and $\left.10 \mathrm{mM} \mathrm{H}_{2} \mathrm{O}_{2}\right), 20 \mu \mathrm{l}$ of total extract $(\sim 22 \mu \mathrm{g}$ protein) or cell wall-bound enriched extract were added. Tetraguaiacol formation (using $\varepsilon=26,600 \mathrm{M}^{-1} \mathrm{~cm}^{-1}$; Chance and Maehly, 1955) was monitored at $470 \mathrm{~nm}$.

Catalase activity was determined (Cakmak and Marschner, 1992) in a reaction mixture comprising $1.5 \mathrm{ml}$ of the reaction buffer (25 mM phosphate buffer - $\mathrm{pH} \mathrm{7.0,} \mathrm{and} 10 \mathrm{mM} \mathrm{H}_{2} \mathrm{O}_{2}$ ) and 
$20 \mu \mathrm{l}$ of total protein extract ( $\sim 22 \mu \mathrm{g}$ protein). $\mathrm{H}_{2} \mathrm{O}_{2}$ decomposition (using $\varepsilon=39.4 \mathrm{M}^{-1} \mathrm{~cm}^{-1}$; Nelson and Kiesow, 1972) was followed at $240 \mathrm{~nm}$.

Superoxide dismutase activity was measured as per Beyer and Fridovich (1987). To $1 \mathrm{ml}$ of the reaction buffer $(50 \mathrm{mM}$ phosphate buffer - pH 7.8, $57 \mu \mathrm{M}$ NBT, $9.9 \mathrm{mM}$ L-methionine, and $0.025 \%$ Triton $\mathrm{X}-100), 10 \mu \mathrm{l}$ of $44 \mathrm{mg} \mathrm{l}^{-1}$ riboflavin and $20 \mu \mathrm{l}$ of total protein extract $(\sim 11 \mu \mathrm{g}$ protein $)$ were added. The reaction took place under white light for $15 \mathrm{~min}$ at room temperature. Final absorbance value was read at $560 \mathrm{~nm}$. One SOD activity unit (U) was defined as the amount of active enzyme needed to inhibit NBT reduction by $50 \%$.

\section{Flavonoid Content}

Flavonoid content was estimated according to Zhishen et al. (1999) with minor modifications. Approximately $40 \mathrm{mg}$ of frozen plant tissue was ground in liquid nitrogen, extracted in $300 \mu \mathrm{l}$ of $95 \%$ ethanol $[\mathrm{v} / \mathrm{v}]$, and placed in an ultrasonic bath for $30 \mathrm{~min}$ at $4^{\circ} \mathrm{C}$ in the dark. The next steps were performed under indirect light. Extracts were centrifuged at $15,000 \times g$ for $10 \mathrm{~min}$ at $4^{\circ} \mathrm{C}$ and $100 \mu \mathrm{l}$ of the supernatant were mixed with $400 \mu \mathrm{l}$ of distilled water and $30 \mu \mathrm{l}$ of $5 \%$ $\mathrm{NaNO}_{2}$ and kept at room temperature for $5 \mathrm{~min}$. Then, $30 \mu \mathrm{l}$ of $1 \% \mathrm{AlCl}_{3}$ were added and vigorously mixed. After $6 \mathrm{~min}$ at room temperature, $200 \mu \mathrm{l}$ of $1 \mathrm{M} \mathrm{NaOH}$ and $240 \mu \mathrm{l}$ of distilled water were added and mixed well. The absorbance was measured at $510 \mathrm{~nm}$ and a standard curve was established using quercetin (Sigma, United States).

\section{Statistical Analysis}

Means were compared by Student's paired $t$-test using GraphPad Prism v8.0 for Windows (GraphPad Software, United States), unless stated otherwise. Data were expressed as mean \pm SEM and statistical significance was set at $p \leq 0.05$.

For principal component analysis (PCA), log-transformed data from three biological replicates for each species $(\mathrm{sp}$.) $\times$ time permutation in respect to nine assessed variables were used. Factors were retained according to the Kaiser criterion, i.e., eigenvalue $>1$. PCA was performed in R 4.0.3 (R Core Team, 2020), using packages "factoextra" (Kassambara and Mundt, 2020), "FactoMineR” (Lê et al., 2008), “corrplot” (Wei and Simko, 2017), and "pca3d" (Weiner, 2020).

\section{RESULTS}

\section{E. grandis Shows Higher Rooting Capacity and Xylem Development When Compared to E. globulus}

As previously reported, E. grandis showed higher rooting capacity when compared to E. globulus (Figure 1A), yielding similar number of roots per microcutting and root system length (Supplementary Figure 2). Freshly cut sections showed an overt difference in xylem size. E. grandis displayed a more pronounced degree of xylem development (Figure 1B), whereas E. globulus appeared somewhat delayed in its development, often showing various degrees of stellate tissue organization (Figure 1D). In fact, after image processing and xylem area quantification, this finding proved to be statistically significant (Figure 1F). As shown in Figure 1D, E. globulus xylem was often restricted to four poles, barely forming a vascular ring, whereas a well-defined ring was the predominant feature in E. grandis microcuttings. Xylem area did not significantly differ between sampling times for either species.
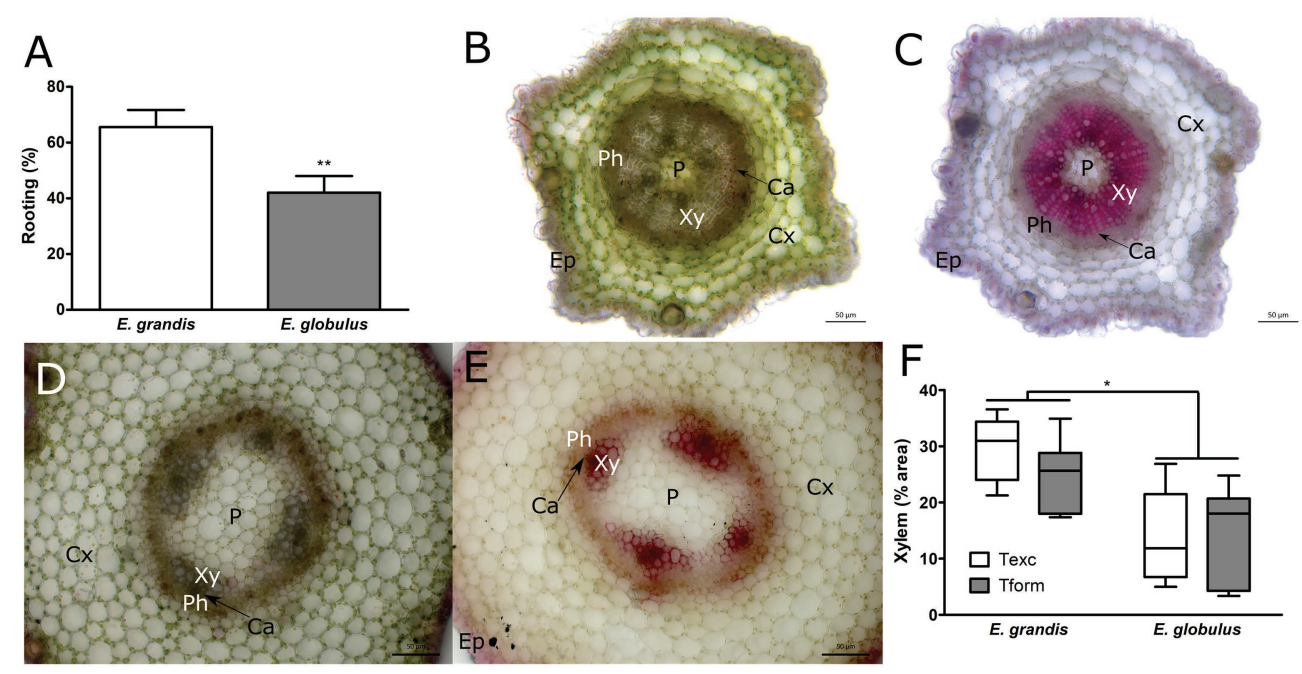

FIGURE 1 | Morphological and developmental patterns of Eucalyptus spp. microcuttings. (A) Rooting capacity (\%) of Eucalyptus grandis and Eucalyptus globulus microcuttings ( $n=60,{ }^{\star \star} p<0.01$, paired $t$-test). Unstained stem transversal sections (B,D) and lignin stained (C,E) of $E$. grandis (B,C) and $E$. globulus (D,E). Images of stem transversal sections were taken at the time of excision (P, pith; Xy, xylem; Ca, cambium; Ph, phloem; Cx, cortex; and Ep, epidermis). (F) Xylem tissue area percentages found in E. grandis and E. globulus transversal stem sections, at the time of excision (Texc) and at the formation stage of adventitious root (AR) development (Tform; $n=12,{ }^{*} p<0.05$, paired $t$-test). Scale bar $=50 \mu \mathrm{m}$. 
Xylem is highly lignified, and E. globulus trees are in the lower end of Eucalyptus spp. lignin content (Nawawi et al., 2017). To investigate if such difference in development could be correlated with lignin content, microcuttings were stained with phloroglucinol (Figures 1C,E). Additionally, whole-cutting lignin content was measured using acetyl bromide method. Phloroglucinol-stained area percentages largely overlapped with xylem area percentages. However, whole-cutting lignin content did not change between species or sampling times (data not shown). At Tform, some AR primordia were forming inside microcutting stems (Supplementary Figure 3).

Both species displayed a range of growth phenotypes, especially since microcuttings were seed-derived, adding variability to the samples. It is worth noting that there was remarkable variation in xylem development, especially in E. globulus. As per Figure 1F, there was some overlap between species, as shown in Figures 2, 3. E. globulus has a higher mean growth rate than E. grandis. Accordingly, previous investigations that used this culture system included a 2-week gap between species (as described in Adventitious rooting section) to obtain more uniform microcuttings. To ensure that differences in xylem area percentage were not a matter of growth, 16-week E. globulus microcuttings were also assessed. Mean xylem area percentage was equal to 14-week E. globulus and inferior to 16-week $E$. grandis (Supplementary Figure 4). This supported the hypothesis that such difference is likely an intrinsic feature of these species.

\section{ROS-Associated Distribution Patterns Vary in E. globulus Along AR Development}

Nitro blue tetrazolium staining was used for histochemical detection of superoxide on stem base sections of E. grandis and E. globulus. At Texc, larger portions of E. globulus microcutting sections were stained with NBT than in E. grandis (Figures 2A,C,E). Conversely, at Tform, very few stained spots could be detected on E. globulus sections (Figure 2D), while no significant difference between sampling times was seen in E. grandis (Figures 2A,B,E). In NBT-stained sections, superoxide anion was most frequently found across the cortical region and, in some cases, more adjacent to the vascular tissues (Figures 2A-D).

To study $\mathrm{H}_{2} \mathrm{O}_{2}$ distribution, E. grandis (Figures 3A,B) and E. globulus (Figures 3C,D) sections were stained with DAB. DAB-stained area did not vary between species at Texc (Figure 3E). E. globulus experienced an increase in $\mathrm{H}_{2} \mathrm{O}_{2}$ distribution from Texc to Tform, especially around the outermost domain of phloem tissue (Figures 3C-E). Unlike superoxide, staining could be observed as a continuum. Though some cells displayed a uniformly stained cytoplasm, DAB precipitate could more intensely be detected along cell walls.

While E. grandis maintained a similar profile at both time points, ROS distribution in E. globulus changed significantly. Superoxide-associated area decreased as $\mathrm{H}_{2} \mathrm{O}_{2}$-associated area increased.

\section{Specific Changes in Redox-Associated Parameters Hint at Divergent Redox- Associated Patterns}

Tissue development and ROS localization are complex phenomena that receive inputs from several genetic and environmental factors. To better characterize the redox status of microcuttings in each AR stage monitored, the activity of some redox-related enzymes was assayed (Figure 4).

Superoxide dismutase activity did not differ between species at Texc, but it decreased with time in E. globulus and at Tform
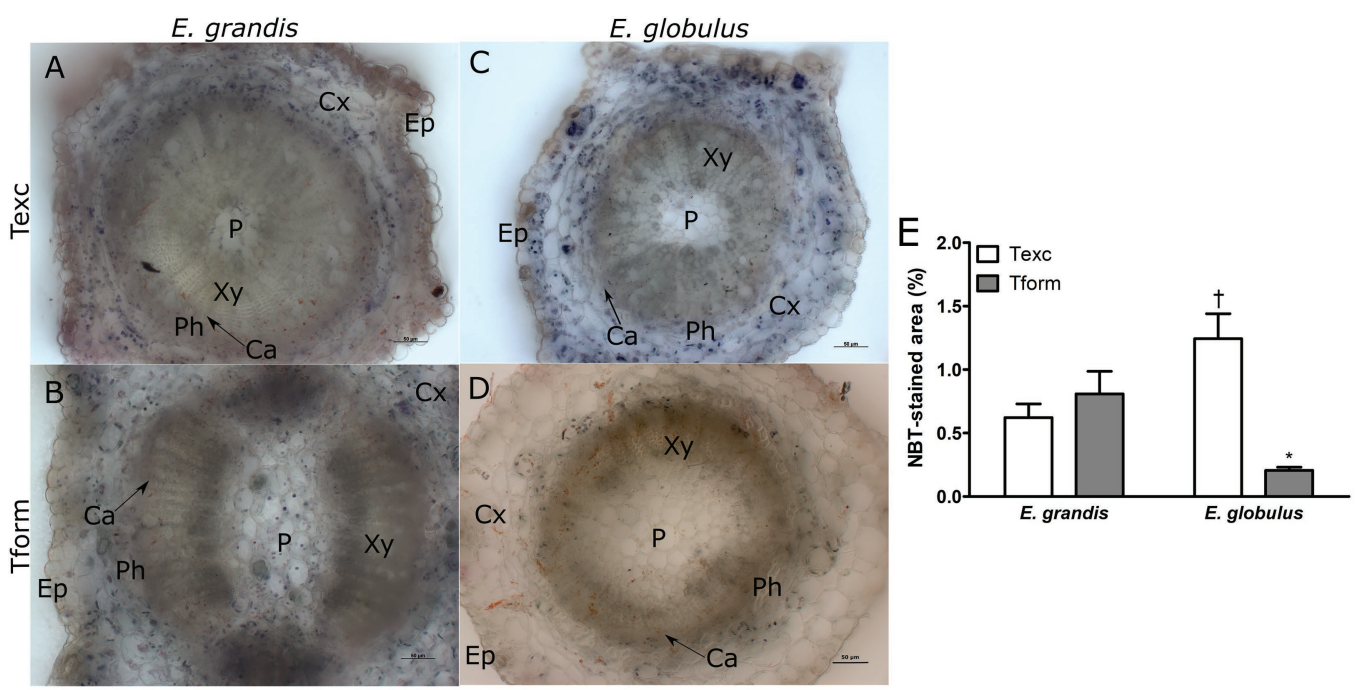

FIGURE 2 | Superoxide localization in Eucalyptus spp. microcutting stem bases. E. grandis (A,B) and E. globulus (C,D) nitro blue tetrazolium (NBT)-stained microcutting transversal stem sections at the time of excision - Texc (A,C) and at the formation stage of AR development - Tform (B,D; P, pith; Xy, xylem; Ca, cambium; Ph, phloem; Cx, cortex; and Ep, epidermis). (E) NBT-stained area percentage in E. grandis and E. globulus transversal stem sections at Texc and Tform ( ${ }^{\star} p<0.05$, paired $t$-test between time points; ${ }^{\dagger} p<0.05$, paired $t$-test between spp.). Scale bar $=50 \mu \mathrm{m}$. 

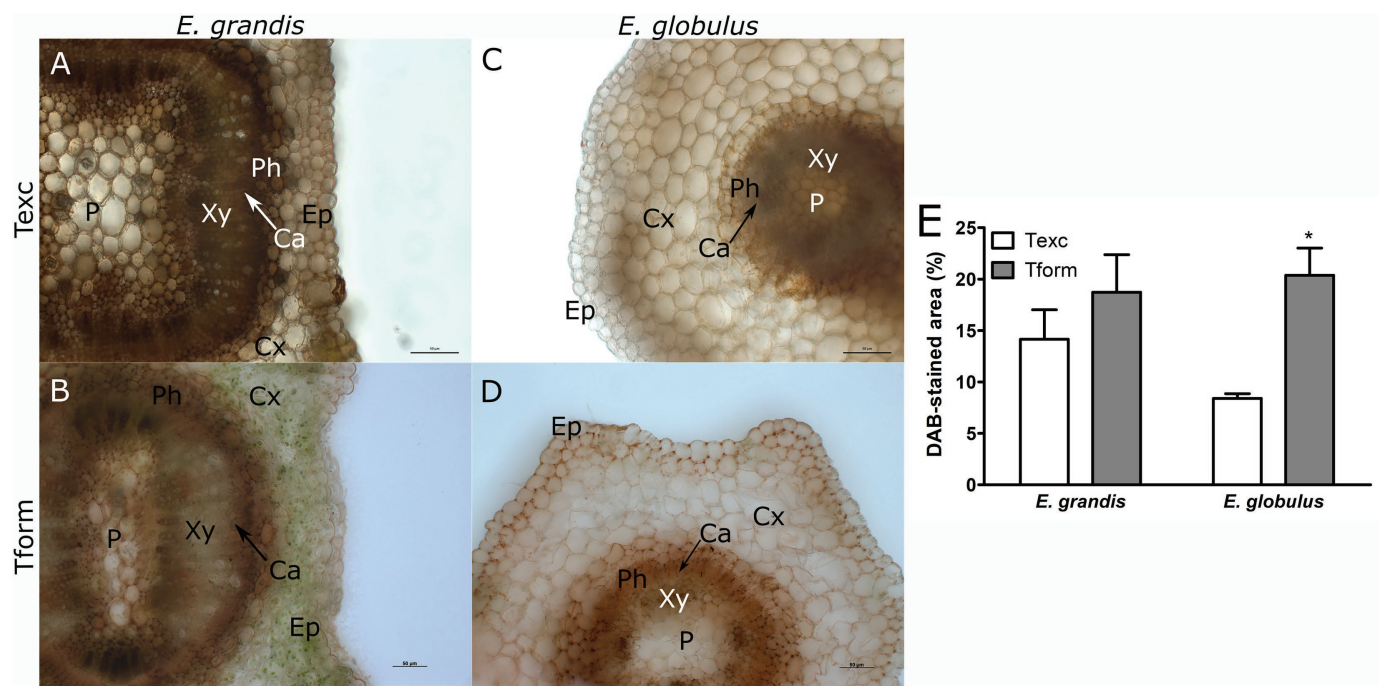

FIGURE 3 | Hydrogen peroxide localization in Eucalyptus spp. microcutting stem bases. E. grandis (A,B) and E. globulus (C,D) microcutting 3,3'-diaminobenzidine (DAB)-stained transversal stem sections at the time of excision - Texc (A,C) and at the formation stage of AR development - Tform (B,D; P, pith; Xy, xylem; Ca, cambium; Ph, phloem; Cx, cortex; and Ep, epidermis). (E) DAB-stained area percentage in $E$. grandis and E. globulus transversal stem sections at Texc and Tform $\left({ }^{*} p<0.05\right.$, paired $t$-test between time points). Scale bar $=50 \mu \mathrm{m}$.
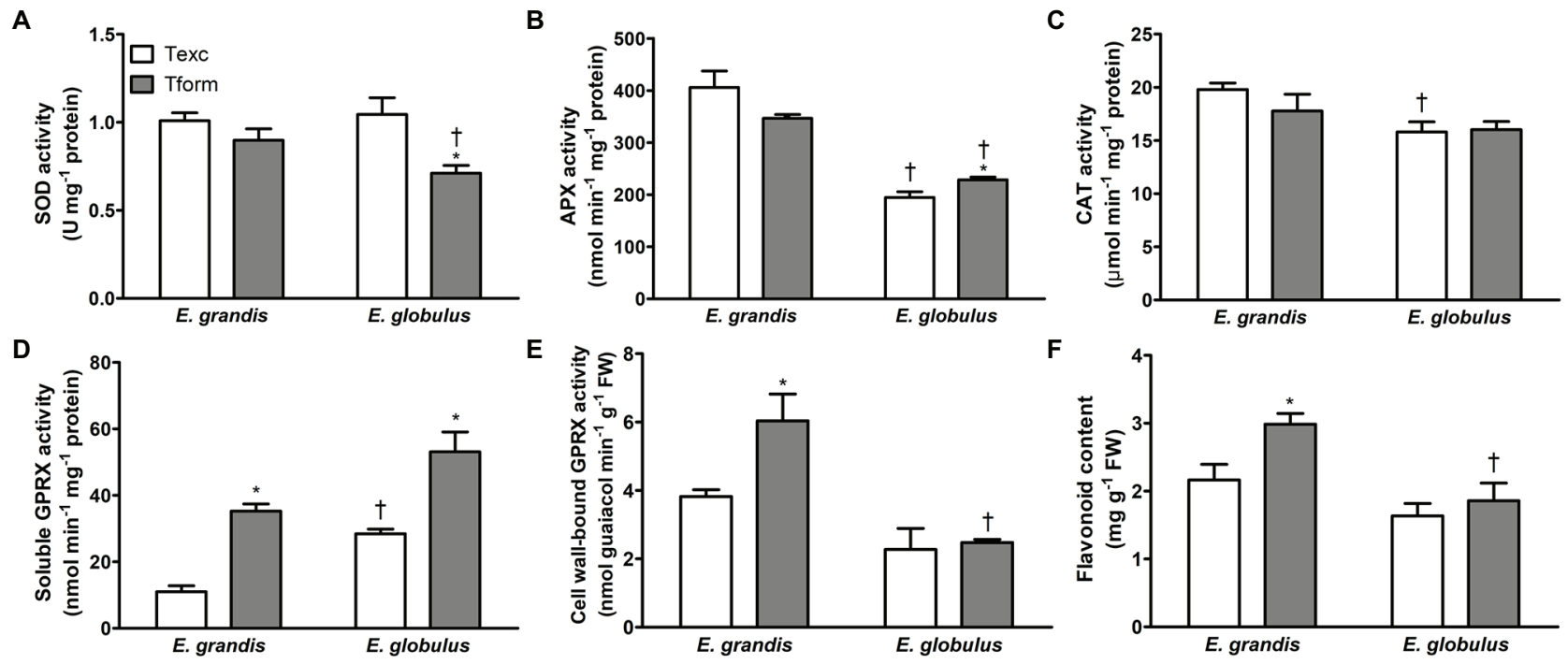

FIGURE 4 | Redox-associated biochemical parameters during adventitious rooting in Eucalyptus spp. microcuttings. Superoxide dismutase (SOD; A), ascorbate peroxidase (APX; B), catalase (CAT; C), soluble (D) and cell wall-bound guaiacol peroxidase (GPRX; E) activity and flavonoid content (F) in E. grandis and E. globulus microcuttings at the time of excision (Texc) and at the formation stage (Tform) of AR development $\left(n=5,{ }^{*} p<0.05\right.$, paired $t$-test between time points; ${ }^{\dagger} p<0.05$, paired $t$-test between spp.).

was lower than in E. grandis (Figure 4A). E. globulus APX activity increased from Texc to Tform, but at both sampling times was inferior to that of E. grandis (Figure 4B). At Texc, CAT activity was lower in E. globulus but did not vary with time in either species (Figure 4C).

Soluble GPRX activity significantly increased between sampling times in both species. At Tform, it was higher in
E. globulus (Figure 4D). Cell wall-bound GPRX activity, conversely, only increased with time in E. grandis, reaching levels higher than those of E. globulus at Tform (Figure 4E). Besides the enzymatic component of microcutting antioxidant defense, flavonoid content was assessed as a potential broadacting ROS quencher (Mierziak et al., 2014). It increased between Texc and Tform in E. grandis, and at the latter time 
point was higher than in E. globulus (Figure 4F). The data clearly indicated varied redox profile between the species.

\section{Principal Component Analysis Identifies Parameters Associated With Species and Rooting Stage}

To better understand where both species (at each time point) stand amid the various quantitative parameters, and if the overall picture may be associated with the divergent rooting capacity, a PCA was performed. Nine variables, i.e., xylem percentage area (Xylem\%), DAB-stained percentage area (DABarea), and NBT-stained percentage area (NBT-area) in histological sections, SOD, APX, CAT, soluble GPRX (SolGPRX), cell wallbound GPRX (CWGPRX) activities, and flavonoid content (flav) in microcuttings were used in the analysis.

Figure 5A shows the loadings plot, with color-coded variables according to $\cos ^{2}$ values (complete version available in Supplementary Figure 5). From $\cos ^{2}$ values and their ratio to the sum of $\cos ^{2}$ values for any given principal component (PC), the contribution (\%) of each variable to each PC can be estimated (Supplementary Figure 6). Besides, how well represented each variable is in either PC, how strongly these datasets are correlated can provide relevant information (Supplementary Figure 7).

"Xylem\%," "SOD," SolGPRX," and "CWGPRX" were the main contributors to PC1. Variance explained by PC2 was mostly made up of "APX," "DAB-area," and "CAT." Interestingly, the variable "flav" split almost evenly between PC1 and PC2. "NBT-area" was not well represented in PC1 and PC2; in fact, it primarily constituted PC3, followed by "SOD" and "SolGPRX." Together, the first three principal components accounted for over $91 \%$ of data variance.
A 3D plot containing data from both species at each time point plotted along the three retained PCs is shown in Figure 5B. Observations derived from three biological replicates of each one of the four $\mathrm{sp}$. $\times$ time permutations clustered by group in different octants altogether. Notably, data from both species at Texc clustered on the positive side of the PC2 axis, though in diagonally opposite quadrants on the PC1 $\times$ PC3 plane. The same occurred for data from both species at Tform, but on the negative side of the PC2 axis. Thus, this dimension appeared to be more associated with the temporal aspect of AR. Each species clustered to either side of PC1. Hence, this axis carries information about what separates E. globulus and E grandis regardless of sampling time. PC3 exhibited what differentiates the states of a species at Texc and at Tform.

Overall, the main variables that explain the distance between E. grandis and E. globulus - irrespective of time - were xylem area percentage, SOD, as well as soluble and cell wall-bound GPRX activity (Supplementary Figure 5). While "SOD," "Xylem\%," "CWGPRX," and "flav" had strong positive correlation with PC1 (and, therefore, with E. grandis phenotype), "SolGPRX" showed strong negative correlation with PC1 (thus associated with E. globulus; Supplementary Figure 7).

Ascorbate peroxidase and CAT activities and DAB-stained area were the main contributors to divergent profiles at Texc and Tform - irrespective of species. PC2 had strong positive correlation with "DAB-area," "CWGPRX," and "flav," and strong negative correlation with "APX" and "CAT." The last two covariates in this dataset and were strongly associated with Tform. Curiously, flavonoid content contributed almost evenly to the differentiation of species and time (Supplementary Figure 6).

Finally, NBT-stained area, SOD, and soluble GPRX activity responded for the smaller fraction of variance that specifically
A

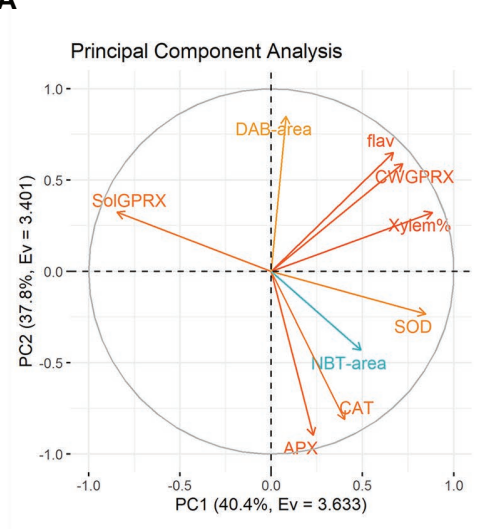

B

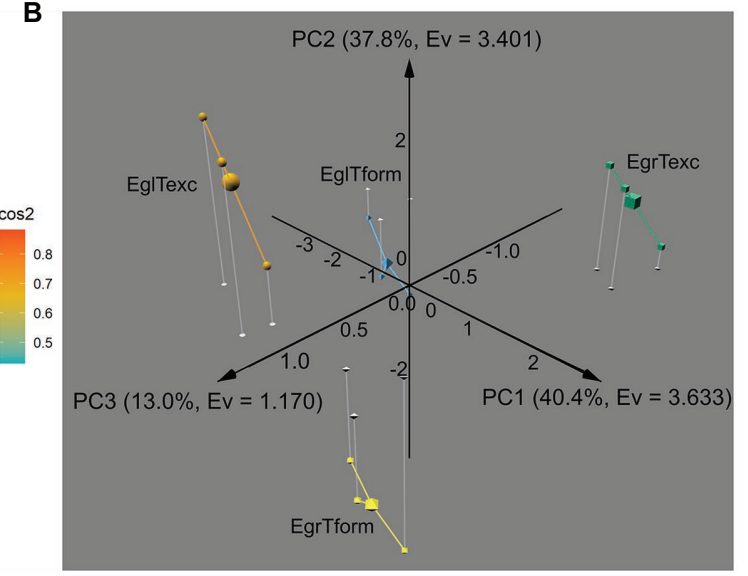

FIGURE 5 | Principal component analysis of histological, histochemical and biochemical parameters assessed during AR development in E. grandis and E. globulus microcuttings. (A) Loadings plot of variables along principal components PC1 and PC2, colored according to cos ${ }^{2}$ values (flav, flavonoid content; CWGPRX, cell wall-bound guaiacol peroxidase activity; Xylem\%, xylem area percentage; SOD, superoxide dismutase activity; NBT-area, nitro blue tetrazoliumstained area percentage; CAT, catalase activity; APX, ascorbate peroxidase activity; SolGPRX, soluble guaiacol peroxidase activity; and DAB-area, 3,3'-diaminobenzidine-stained area percentage). (B) Individual position on a 3D plot along axes PC1, PC2 and PC3 of E. grandis and E. globulus three biological replicates (and each corresponded centroid, represented as a larger solid) at the time of excision -Texc (green cubes and orange spheres, respectively) and at the formation stage of AR development -Tform (yellow octahedrons and blue pyramids, respectively). Principal components are expressed as PC [\%variance, eigenvalue (Ev)]. EgrTexc, E. grandis at Texc; EgrTform, E. grandis at Tform; EglTexc, E. globulus at Texc; and EglTform, E. globulus at Tform. 
defined each species at each time point. "NBT-area" was the only variable strongly correlated, i.e., correlation coefficient absolute value $<0.5$, with PC3. Though most of the variance (over $78 \%$ ) was explained by PC1 $(40.4 \%$, a proxy for the "species" factor) and PC2 (37.8\% - associated with the "time" factor), a notable $13 \%$ constituted sp. $\times$ time factor interactionderived variance. Out of the nine parameters entered in the PCA, NBT-stained area percentage almost single-handedly represented the interaction.

\section{DISCUSSION}

Greater presence of vascular tissue was observed in E. grandis microcuttings when compared to E. globulus (Figures 1B-F). This may indicate delayed vascular tissue differentiation of the latter species in relation to the former. Such disparity in xylem area percentage between the two species, though not the main focus of their work, can be also observed in the data of Bryant and Trueman (2015). Also, the primitive state of vascular tissue development observed in E. globulus, with interfascicular cambium and stellate vascular bundle pattern (as seen in Figures 1D,E), has been previously reported (Baltierra et al., 2004). A withinspecies anatomical comparison of E. grandis $\times E$. urophylla clones with varying rooting capacity, all of which were above $80 \%$, did not reveal an association between xylem area percentage and rooting competence, although overall cutting anatomy was consistent with the patterns found in this study (Goulart et al., 2014). A general panel of Eucalyptus spp. rooting ability, tissue proportion, and auxin content, though elusive at this time, could help explain the variability of these traits across the genus and perhaps show phylogenetic or biogeographical links.

The increased xylem development observed in E. grandis when compared to E. globulus (Figure 1F) could be related to differences in auxin and ethylene production between these species. De Almeida et al. (2015) examined cambium-specific gene expression and auxin localization during AR development, finding that in vitro grown E. globulus had lower endogenous auxin content than E. grandis. Besides being a crucial regulator of AR formation, auxin is involved in xylem development. Overexpression of an impaired version of Populus tomentosa AUXIN/INDOLE-3-ACETIC ACID (PtoIAA9), an auxin-response module related to wood formation, led to lower xylem area percentages (Xu et al., 2019). Auxin has been shown to induce xylem differentiation through LONESOME HIGHWAY-LIKE 3 in Arabidopsis (Ohashi-Ito et al., 2013). Along with gibberellin, it has been reported as a regulator of transcriptional changes needed for xylem differentiation and secondary cell wall deposition. Auxin treatment of poplar and Arabidopsis led to higher xylem differentiation and reduced secondary cell wall thickness (Johnsson et al., 2019). Yet, overexpression of YUCCA genes, which encode enzymes on the auxin biosynthesis pathway, led to an increase in phloroglucinol-stained area, perhaps due to increased ethylene production and signaling (Hentrich et al., 2013). Several auxin analogs, when applied to Arabidopsis, caused an increase in xylem development rates (Yoshimoto et al., 2012). E. grandis, known to have higher basal auxin levels, may be more prone to xylem differentiation. The same species also had lower expression levels of an Arabidopsis IAA12 homolog, known as a suppressor of auxin signaling (De Almeida et al., 2015). How different durations of auxin treatment could affect xylem development and lignin distribution patterns remains an outstanding question.

Phloroglucinol-stained area, largely correspondent to highly lignified xylem tissue (Figures 1C,E), was more prominently present in E. grandis microcuttings. However, whole-microcutting lignin content did not differ between species. Lignin content is, besides a physiologically interesting parameter, an industrially relevant trait in Eucalyptus trees. Although high levels of specific lignins may improve wood pyrolysis for charcoal production (de Paula Protásio et al., 2020), kraft pulping benefits significantly from low lignin content (Pilate et al., 2002). Lignin is assembled in cell walls from phenolic units by a process that naturally involves ROS generation and peroxidase activity (Tobimatsu and Schuetz, 2019).

Multivariate analysis has shown E. grandis and E. globulus to diverge in respect to redox-associated parameters in both assessed time points (Figure 5). In fact, antioxidant enzyme activity was among the main factors responsible for such different profiles (Supplementary Figure 6). ROS play vital signaling roles in plant development, often in an organ-, tissue-, cell-, and even organelle-specific manner (Mhamdi and Van Breusegem, 2018). Undoubtedly, measurement of specific reactive species can help understand global changes in the ROS pool. Still, histochemical localization of these substances, while restricted to each tissue section, may provide another dimension of understanding developmental processes. This is especially the case for a microcutting, in which a metabolic gradient is created by the excision itself (Druege et al., 2019).

Microcutting staining revealed tissue-localized patterns for superoxide and hydrogen peroxide in both species (Figures 2, 3). Superoxide anion has a very short half-life. Its unstable and radical chemical character means it will most likely attack the nearest compatible molecule within reach, i.e., Fe-S proteins, to achieve a more favorable state (Mittler, 2017). Regarding superoxide roles in AR development, Tewari et al. (2008) suggested its participation in nitric oxide (NO)-mediated adventitious rooting of Asian ginseng. Moreover, in cucumber cuttings under osmotic stress, superoxide has been shown to increase root number per explant when coupled with NO (Niu et al., 2017).

Hydrogen peroxide, in contrast to superoxide, is a non-radical ROS with a somewhat longer half-life. It is crucial for lignin polymerization reactions in cell walls. It is also directly or indirectly involved in senescence, responsiveness to stress, phytohormone homeostasis, and metabolic modulation (Petrov and Van Breusegem, 2012). It can be both a product of superoxide detoxification and a source of new, more toxic ROS, through Fenton chemistry. In fact, plant growth itself, relying on highly energetic reactions of photosystem and electron transport chain function, generates more ROS (Noctor et al., 2018; Khorobrykh et al., 2020).

Most of the redox-associated parameters assessed in this study could modulate $\mathrm{H}_{2} \mathrm{O}_{2}$ concentration. If $\mathrm{DAB}$-stained area measurements are interpreted as the sum of all these forces, $\mathrm{H}_{2} \mathrm{O}_{2}$ presence in microcutting stem bases (Figure 3 ) is correlated 
with changes between Texc and Tform for both species. DAB-stained areas seemed to be associated with rapid growth tissues, such as primary xylem, phloem and epidermis. $\mathrm{H}_{2} \mathrm{O}_{2}$ has been shown to stimulate AR formation in mung bean (Li et al., 2009; Huang et al., 2011), ground-cover chrysanthemum (Liao et al., 2010), and marigold (Liao et al., 2011) in a dosedependent manner. It was observed that $\mathrm{H}_{2} \mathrm{O}_{2}$ concentrations increase with time after excision in mung bean hypocotyl cuttings and that ascorbate, a reducing substrate for $\mathrm{H}_{2} \mathrm{O}_{2}$ removal, prevented AR induction (Huang et al., 2011). In poplar, optimal concentrations of $\mathrm{H}_{2} \mathrm{O}_{2}$ were able to accelerate AR formation, whereas higher concentrations slowed down this process and reduced root growth (Zhang et al., 2019a). ROS-mediated regulation of AR development entails auxin participation. The existence of feedback loops between auxin biosynthesis, transport, and signaling and ROS signaling has been suggested (Steffens and Rasmussen, 2016). Application of the auxin indole-3-butyric acid (IBA) promoted $\mathrm{H}_{2} \mathrm{O}_{2}$ production in mung bean seedlings (Li et al., 2009). Wounding induced ROS-modulated auxin biosynthesis- and transportrelated genes, leading to accumulation of this phytohormone in the base of Arabidopsis cuttings (Huang et al., 2020).

Although broadly employed, NBT- and DAB-staining have some limitations regarding specificity, and should be viewed with caution. NBT is labile upon facing a range of molecules besides superoxide, and the exposure of its reduced form to $\mathrm{O}_{2}$ can regenerate NBT. DAB precipitation may occur due to higher peroxidase activity (Noctor et al., 2016). Therefore, their examination together with a pool of related parameters, such as antioxidant defenses, is advisable.

Interplay between auxin and ROS has been the focus of several studies. One of the many effects of auxin treatment is an increase in ROS generation. Moreover, auxin signaling attenuation has been shown to be affected by high levels of ROS. RAPID OXIDATIVE BURST HOMOLOG D (RBOHD) has been associated with this phenomenon, since rbohD Arabidopsis mutants accumulate ROS to a lesser extent (Peer and Murphy, 2013).

Upon cutting excision, a torrent of ROS is locally generated in response to tissue damage. Wound-response in cuttings is mediated by jasmonate (JA; Druege et al., 2016; Zhang et al., 2019b), and JA-mediated wound response has been associated with increased antioxidant enzyme activity (Dar et al., 2015). Such wound-induced oxidative burst has been associated with both RBOHD and peroxidase activity, and the use of enzyme inhibitors impaired AR formation in Arabidopsis. Also essential to ROS propagation and proper signaling were cell wall-bound peroxidases of hypocotyls (Huang et al., 2020). The present study indicated that both cell wall-bound and soluble GPRX activities strongly correlated with rooting phenotype, the former positively while the latter negatively (Figures $4 \mathrm{D}, \mathrm{E}$ ). This may suggest a role for the cell wall-bound fraction in signal propagation rather than as an antioxidant.

Soluble peroxidase activity increased with age in E. globulus microcuttings, the opposite occurring with rooting capacity and auxin content (Aumond et al., 2017). Cucumber cuttings under osmotic stress treated with the NO source sodium nitroprusside experienced higher SOD, CAT, and APX activity, which was associated with improved rooting (Niu et al., 2017). Soluble peroxidase activity induction during rooting of Passiflora suberosa cuttings led to lower rooting percentages (Vilasboa et al., 2020). In the current study, PCA revealed SOD activity to be strongly correlated with $E$. grandis, the easy-to-root species. Furthermore, APX and CAT activities co-variated and were associated with the formation stage of AR development. A comparison of E. globulus and E. grandis microcutting proteomic profiles revealed a substantial portion $(22 \%)$ of differently abundant proteins to be redox-related. Patterns also reflected changes in proteomic profiles from the induction to the formation stage of AR development (De Almeida et al., 2020).

Nonenzymatic antioxidants have also been implicated in auxin-ROS crosstalk. Among these, flavonoids stand out for their multiple interactions with factors that play roles in this scenario. These molecules are known to interfere with auxin transport and thought to mitigate local ROS accumulation that could hinder auxin homeostasis (Peer and Murphy, 2007). The main auxin oxidation product was found at lower concentrations in transparent testa 3 (tt3) Arabidopsis mutants, which accumulate flavonols, whereas flavonoid-deficient $t t 4$ mutants had higher concentrations of the same catabolite. This indicates a possible role for flavonoids as ROS-induced auxin degradation buffers (Peer and Murphy, 2013). In a comparative study of two olive cultivars of different rooting capacity, strong positive correlation was found between total flavonoid content and rooting percentage (Denaxa et al., 2020). An increase in E. gunnii cutting flavonoid content has been associated with overcoming of AR recalcitrance (Di Battista et al., 2019). In the present work, the flavonoid content parameter was virtually equally represented in the "species" and "time" factors. Such diffuse response, unique in this dataset, hints at genotype- and developmental timing-related metabolic differences between the two species. These details, as is the case of many of the whole-microcutting analyses undertaken here, may be further explored by means of a detailed profiling of the stem base and its different tissues.

\section{CONCLUSION}

As a first incursion into the complexity of the comparative redox status during Eucalyptus spp. adventitious rooting, the data from this study revealed different profiles, explained mainly by species- and time-linked variations. Eucalyptus grandis microcuttings showed larger xylem area than E. globulus. Along with this feature, SOD and both cell-wall bound and soluble GPRX activities were indicative of rooting capacity. DAB-stained area, as well as APX and CAT activities, were associated with changes from cutting excision to the AR formation stage, regardless of species. Flavonoid content was associated with both time- and species-derived variation. Overall, data indicate that E. grandis shows higher cambium activity and tighter modulation of redox conditions than E. globulus. These features may influence ROS-based signaling and phytohormone homeostasis of cuttings, thereby impacting on AR development. Besides being players in the realm of AR developmental 
differences, the specific features herein identified could become potential tools for early clone selection and AR modulation aiming at improved clonal propagation of this forest crop.

Future studies of AR recalcitrance will be able to further explore parameters that correlate with rooting capacity and add elements such as phytohormones and additional antioxidants, as well as other Eucalyptus spp. and their hybrids across the spectrum of rooting capacity. If validated under commercial greenhouse production settings and across several genotypes, some of the parameters explored in this study may contribute to the establishment of robust global models for early prediction of rooting recalcitrance, thereby contributing to germplasm selection and clonal propagation programs.

\section{DATA AVAILABILITY STATEMENT}

The original contributions presented in the study are included in the article/Supplementary Material, further inquiries can be directed to the corresponding author.

\section{AUTHOR CONTRIBUTIONS}

JV, CC, and AF-N contributed to conception and design of the study. JV, CC, and LR set up the in vitro culture system and performed biochemical analyses. JV, CC, and JM sectioned,

\section{REFERENCES}

Anjum, N. A., Sharma, P., Gill, S. S., Hasanuzzaman, M., Khan, E. A., Kachhap, K., et al. (2016). Catalase and ascorbate peroxidase-representative $\mathrm{H}_{2} \mathrm{O}_{2}$ detoxifying heme enzymes in plants. Environ. Sci. Pollut. Res. 23, 19002-19029. doi: 10.1007/s11356-016-7309-6

Aumond, M. L., de Araujo, A. T., de Oliveira Junkes, C. F., de Almeida, M. R., Matsuura, H. N., de Costa, F., et al. (2017). Events associated with early age-related decline in adventitious rooting competence of Eucalyptus globulus Labill. Front. Plant Sci. 8:1734. doi: 10.3389/fpls.2017.01734

Baltierra, X. C., Montenegro, G., and De García, E. (2004). Ontogeny of in vitro rooting processes in Eucalyptus globulus. In Vitro Cell. Dev-Pl. 40, 499-503. doi: 10.1079/IVP2004559

Bellini, C., Pacurar, D. I., and Perrone, I. (2014). Adventitious roots and lateral loots: similarities and differences. Annu. Rev. Plant Biol. 65, 639-666. doi: 10.1146/annurev-arplant-050213-035645

Beyer, W. F., and Fridovich, I. (1987). Assaying for superoxide dismutase activity: some large consequences of minor changes in conditions. Anal. Biochem. 161, 559-566. doi: 10.1016/0003-2697(87)90489-1

Bradford, M. M. (1976). A rapid and sensitive method for the quantitation of microgram quantities of protein utilizing the principle of protein-dye binding. Anal. Biochem. 72, 248-254. doi: 10.1016/0003-2697(76)90527-3

Bryant, P. H., and Trueman, S. J. (2015). Stem anatomy and adventitious root formation in cuttings of Angophora, Corymbia and Eucalyptus. Forests 6, 1227-1238. doi: 10.3390/f6041227

Cakmak, I., and Marschner, H. (1992). Magnesium deficiency and high light intensity enhance activities of superoxide dismutase, ascorbate peroxidase, and glutathione reductase in bean leaves. Plant Physiol. 98, 1222-1227. doi: 10.1104/pp.98.4.1222

Carrillo, I., Vidal, C., Elissetche, J. P., and Mendonça, R. T. (2018). Wood anatomical and chemical properties related to the pulpability of Eucalyptus globulus: a review. South. For. 80, 1-8. doi: 10.2989/20702620.2016.1274859

Chance, B., and Maehly, A. C. (1955). Assay of catalases and peroxidases. Methods Enzymol. 2, 764-775. doi: 10.1016/S0076-6879(55)02300-8 stained, and imaged microcuttings. JV performed the statistical analysis. CC and JV wrote the first draft of the manuscript. AF-N reviewed and finalized the manuscript, and warranted financial support. All authors contributed to manuscript revision, read, and approved the submitted version.

\section{FUNDING}

This work was supported by the Conselho Nacional de Desenvolvimento Científico e Tecnológico (CNPq - Brazil, grant 303560/2017-7) and the Coordenação de Aperfeiçoamento de Pessoal de Nível Superior - (CAPES - Brazil) - Finance Code 001.

\section{ACKNOWLEDGMENTS}

We thank Juliana Troleis and Juciana C. Cazarolli (Institute of Biosciences, UFRGS) for technical support.

\section{SUPPLEMENTARY MATERIAL}

The Supplementary Material for this article can be found online at: https://www.frontiersin.org/articles/10.3389/fpls.2020.620832/ full\#supplementary-material

Correa-Aragunde, N., Foresi, N., and Lamattina, L. (2016). "Auxin and nitric oxide: a counterbalanced partnership ensures the redox cue control required for determining root growth pattern" in Advances in Botanical Research. Vol. 77. ed. D. Wendehenne (Cambridge, MA: Academic Press), 41-54.

Da Costa, C. T., de Almeida, M. R., Ruedell, C. M., Schwambach, J., Maraschin, F. S., and Fett-Neto, A. G. (2013). When stress and development go hand in hand: main hormonal controls of adventitious rooting in cuttings. Front. Plant Sci. 4:133. doi: 10.3389/fpls.2013.00133

Dar, T. A., Uddin, M., Khan, M. M. A., Hakeem, K. R., and Jaleel, H. (2015). Jasmonates counter plant stress: a review. Environ. Exp. Bot. 115, 49-57. doi: 10.1016/j.envexpbot.2015.02.010

De Almeida, M. R., de Bastiani, D., Gaeta, M. L., de Araújo Mariath, J. E., de Costa, F., Retallick, J., et al. (2015). Comparative transcriptional analysis provides new insights into the molecular basis of adventitious rooting recalcitrance in Eucalyptus. Plant Sci. 239, 155-165. doi: 10.1016/j.plantsci.2015.07.022

De Almeida, M. R., Schwambach, J., Silveira, V., Heringer, A. S., Fett, J. P., and Fett-Neto, A. G. (2020). Proteomic profiles during adventitious rooting of Eucalyptus species relevant to the cellulose industry. New For. 51, 213-241. doi: 10.1007/s11056-019-09728-7

de Paula Protásio, T., Lima, M. D. R., Teixeira, R. A. C., do Rosário, F. S., de Araújo, A. C. C., de Assis, M. R., et al. (2020). Influence of extractives content and lignin quality of Eucalyptus wood in the mass balance of pyrolysis process. Bioenergy Res. 1-15. doi: 10.1007/s12155-020-10166-Z

Denaxa, N. K., Roussos, P. A., and Vemmos, S. N. (2020). Assigning a role to the endogenous phenolic compounds on adventitious root formation of olive stem cuttings. J. Plant Growth Regul. 39, 411-421. doi: 10.1007/ s00344-019-09991-0

Di Battista, F., Maccario, D., Beruto, M., Grauso, L., Lanzotti, V., Curir, P., et al. (2019). Metabolic changes associated to the unblocking of adventitious root formation in aged, rooting-recalcitrant cuttings of Eucalyptus gunnii Hook. f. (Myrtaceae). Plant Growth Regul. 89, 73-82. doi: 10.1007/ s10725-019-00515-0

Dos Santos, W. D., Ferrarese, M. D. L. L., Finger, A., Teixeira, A. C. N., and Ferrarese-Filho, O. (2004). Lignification and related enzymes in Glycine max 
root growth-inhibition by ferulic acid. J. Chem. Ecol. 30, 1203-1212. doi: 10.1023/B:JOEC.0000030272.83794.f0

Druege, U., Franken, P., and Hajirezaei, M. R. (2016). Plant hormone homeostasis, signaling, and function during adventitious root formation in cuttings. Front. Plant Sci. 7:381. doi: 10.3389/fpls.2016.00381

Druege, U., Hilo, A., Pérez-Pérez, J. M., Klopotek, Y., Acosta, M., Shahinnia, F., et al. (2019). Molecular and physiological control of adventitious rooting in cuttings: phytohormone action meets resource allocation. Ann. Bot. 123, 929-949. doi: $10.1093 / \mathrm{aob} / \mathrm{mcy} 234$

Fett-Neto, A. G., Fett, J. P., Vieira Goulart, L. W., Pasquali, G., Termignoni, R. R., and Ferreira, A. G. (2001). Distinct effects of auxin and light on adventitious root development in Eucalyptus saligna and Eucalyptus globulus. Tree Physiol. 21, 457-464. doi: 10.1093/treephys/21.7.457

Gechev, T. S., Van Breusegem, F., Stone, J. M., Denev, I., and Laloi, C. (2006). Reactive oxygen species as signals that modulate plant stress responses and programmed cell death. BioEssays 28, 1091-1101. doi: 10.1002/bies.20493

Goulart, P. B., Xavier, A., Iarema, L., and Otoni, W. C. (2014). Morpho-anatomy of adventitious rhizogenesis in mini-cuttings of Eucalyptus grandis $\mathrm{x}$ Eucalyptus urophylla. Ciênc. Florest. 24, 521-532. doi: 10.1590/1980-509820142403001

Hentrich, M., Sánchez-Parra, B., Marta-Marina, P. A., Loba, V. C., Carrillo, L., Vicente-Carbajosa, J., et al. (2013). YUCCA8 and YUCCA9 overexpression reveals a link between auxin signaling and lignification through the induction of ethylene biosynthesis. Plant Signal. Behav. 8:e26363. doi: 10.4161/psb.26363

Huang, A. X., She, X. P., Cao, B. H., and Ren, Y. (2011). Distribution of hydrogen peroxide during adventitious roots initiation and development in mung bean hypocotyls cuttings. Plant Growth Regul. 64, 109-118. doi: 10.1007/s10725-010-9545-y

Huang, A., Wang, Y., Liu, Y., Wang, G., and She, X. (2020). Reactive oxygen species regulate auxin levels to mediate adventitious root induction in Arabidopsis hypocotyl cuttings. J. Integr. Plant Biol. 62, 912-926. doi: 10.1111/ jipb. 12870

Johansen, D. A. (1940). Plant microtechniques. New York: McGraw-Hill Book Co. Ink, 523.

Johnsson, C., Jin, X., Xue, W., Dubreuil, C., Lezhneva, L., and Fischer, U. (2019). The plant hormone auxin directs timing of xylem development by inhibition of secondary cell wall deposition through repression of secondary wall NAC-domain transcription factors. Physiol. Plant. 165, 673-689. doi: $10.1111 /$ ppl.12766

Kapoor, D., Singh, S., Kumar, V., Romero, R., Prasad, R., and Singh, J. (2019). Antioxidant enzymes regulation in plants in reference to reactive oxygen species (ROS) and reactive nitrogen species (RNS). Plant Gene 19:100182. doi: $10.1016 /$ j.plgene.2019.100182

Kassambara, A., and Mundt, F. (2020). factoextra: extract and visualize the results of multivariate data analyses. $\mathrm{R}$ package version 1.0.7. Available at: https://CRAN.R-project.org/package=factoextra (Accessed October 10, 2020).

Khorobrykh, S., Havurinne, V., Mattila, H., and Tyystjärvi, E. (2020). Oxygen and ROS in photosynthesis. Plan. Theory 9:91. doi: 10.3390/plants9010091

Lê, S., Josse, J., and Husson, F. (2008). FactoMineR: An R package for multivariate analysis. J. Stat. Softw. 25, 1-18. doi: 10.18637/jss.v025.i01

Li, S. W., Xue, L., Xu, S., Feng, H., and An, L. (2009). Hydrogen peroxide acts as a signal molecule in the adventitious root formation of mung bean seedlings. Environ. Exp. Bot. 65, 63-71. doi: 10.1016/j.envexpbot.2008.06.004

Liao, W., Huang, G., Yu, J., Zhang, M., and Shi, X. (2011). Nitric oxide and hydrogen peroxide are involved in indole- 3 butyric acid-induced adventitious root development in marigold. J. Hortic. Sci. Biotechnol. 86, 159-165. doi: 10.1080/14620316.2011.11512742

Liao, W. B., Xiao, H. L., and Zhang, M. L. (2010). Effect of nitric oxide and hydrogen peroxide on adventitious root development from cuttings of groundcover chrysanthemum and associated biochemical changes. J. Plant Growth Regul. 29, 338-348. doi: 10.1007/s00344-010-9140-5

McDowell, E. M., and Trump, B. F. (1976). Histologic fixatives suitable for diagnostic light and electron microscopy. Arch. Pathol. Lab. Med. 100, 405-414.

Mhamdi, A., and Van Breusegem, F. (2018). Reactive oxygen species in plant development. Development 145:dev164376. doi: 10.1242/dev.164376

Mierziak, J., Kostyn, K., and Kulma, A. (2014). Flavonoids as important molecules of plant interactions with the environment. Molecules 19, 16240-16265. doi: 10.3390/molecules 191016240
Mittler, R. (2017). ROS are good. Trends Plant Sci. 22, 11-19. doi: 10.1016/j. tplants.2016.08.002

Mittler, R., Vanderauwera, S., Gollery, M., and Van Breusegem, F. (2004). Reactive oxygen gene network of plants. Trends Plant Sci. 9, 490-498. doi: 10.1016/j.tplants.2004.08.009

Mittler, R., Vanderauwera, S., Suzuki, N., Miller, G., Tognetti, V. B., Vandepoele, K., et al. (2011). ROS signaling: the new wave? Trends Plant Sci. 16, 300-309. doi: $10.1016 /$ j.tplants.2011.03.007

Murashige, T., and Skoog, F. (1962). A revised medium for rapid growth and bio assays with tobacco tissue cultures. Physiol. Plant. 15, 473-497. doi: 10.1111/j.1399-3054.1962.tb08052.x

Nakano, Y., and Asada, K. (1981). Hydrogen peroxide is scavenged by ascorbatespecific peroxidase in spinach chloroplasts. Plant Cell Physiol. 22, 867-880 doi: 10.1093/oxfordjournals.pcp.a076232

Nawawi, D. S., Syafii, W., Tomoda, I., Uchida, Y., Akiyama, T., Yokoyama, T., et al. (2017). Characteristics and reactivity of lignin in Acacia and Eucalyptus woods. J. Wood Chem. Technol. 37, 273-282. doi: 10.1080/ 02773813.2017.1291684

Nelson, D. P., and Kiesow, L. A. (1972). Enthalpy of decomposition of hydrogen peroxide by catalase at $25^{\circ} \mathrm{C}$ (with molar extinction coefficients of $\mathrm{H}_{2} \mathrm{O}_{2}$ solutions in the UV). Anal. Biochem. 49, 474-478. doi: 10.1016/ 0003-2697(72)90451-4

Niu, L., Yu, J., Liao, W., Yu, J., Zhang, M., and Dawuda, M. M. (2017). Calcium and calmodulin are involved in nitric oxide-induced adventitious rooting of cucumber under simulated osmotic stress. Front. Plant Sci. 8:1684. doi: 10.3389/fpls.2017.01684

Noctor, G., Mhamdi, A., and Foyer, C. H. (2016). Oxidative stress and antioxidative systems: recipes for successful data collection and interpretation. Plant Cell Environ. 39, 1140-1160. doi: 10.1111/pce.12726

Noctor, G., Reichheld, J. P., and Foyer, C. H. (2018). ROS-related redox regulation and signaling in plants. Semin. Cell Dev. Biol. 80, 3-12. doi: 10.1016/j. semcdb.2017.07.013

Ohashi-Ito, K., Matsukawa, M., and Fukuda, H. (2013). An atypical bHLH transcription factor regulates early xylem development downstream of auxin. Plant Cell Physiol. 54, 398-405. doi: 10.1093/pcp/pct013

Pacurar, D. I., Perrone, I., and Bellini, C. (2014). Auxin is a central player in the hormone cross-talks that control adventitious rooting. Physiol. Plant. 151, 83-96. doi: 10.1111/ppl.12171

Peer, W. A., and Murphy, A. S. (2007). Flavonoids and auxin transport: modulators or regulators? Trends Plant Sci. 12, 556-563. doi: 10.1016/j.tplants.2007.10.003

Peer, W. A., and Murphy, A. S. (2013). Evidence of oxidative attenuation of auxin signalling. J. Exp. Bot. 64, 2629-2639. doi: 10.1093/jxb/ert152

Penín, L., López, M., Santos, V., Alonso, J. L., and Parajó, J. C. (2020). Technologies for Eucalyptus wood processing in the scope of biorefineries: a comprehensive review. Bioresour. Technol. 311:123528. doi: 10.1016/j.biortech.2020.123528

Petrov, V. D., and Van Breusegem, F. (2012). Hydrogen peroxide-a central hub for information flow in plant cells. AoB Plants 12, 1-13. doi: 10.1093/ aobpla/pls014

Pilate, G., Guiney, E., Holt, K., Petit-Conil, M., Lapierre, C., Leplé, J. C., et al. (2002). Field and pulping performances of transgenic trees with altered lignification. Nat. Biotechnol. 20, 607-612. doi: 10.1038/nbt0602-607

R Core Team (2020). R: A language and environment for statistical computing. R Foundation for Statistical Computing, Vienna, Austria. Available at: https:// www.R-project.org/ (Accessed October 10, 2020).

Rencoret, J., Gutiérrez, A., and Del Río, J. C. (2007). Lipid and lignin composition of woods from different eucalypt species. Holzforschung 61, 165-174. doi 10.1515/HF.2007.030

Romero-Puertas, M. C., Rodriguez-Serrano, M., Corpas, F. J., Gomez, M., Del Rio, L. A., and Sandalio, L. M. (2004). Cadmium-induced subcellular accumulation of $\mathrm{O}_{2}{ }^{-}$and $\mathrm{H}_{2} \mathrm{O}_{2}$ in pea leaves. Plant Cell Environ. 27, 1122-1134. doi: $10.1111 /$ j.1365-3040.2004.01217.x

Rueden, C. T., Schindelin, J., Hiner, M. C., DeZonia, B. E., Walter, A. E., Arena, E. T., et al. (2017). ImageJ2: ImageJ for the next generation of scientific image data. BMC Bioinform. 18:529. doi: 10.1186/s12859-017-1934-z

Schindelin, J., Arganda-Carreras, I., Frise, E., Kaynig, V., Longair, M., Pietzsch, T., et al. (2012). Fiji: An open-source platform for biological-image analysis. Nat. Methods 9, 676-682. doi: 10.1038/nmeth.2019

Steffens, B., and Rasmussen, A. (2016). The physiology of adventitious roots. Plant Physiol. 170, 603-617. doi: 10.1104/pp.15.01360 
Tewari, R. K., Hahn, E. J., and Paek, K. Y. (2008). Function of nitric oxide and superoxide anion in the adventitious root development and antioxidant defence in Panax ginseng. Plant Cell Rep. 27, 563-573. doi: 10.1007/ s00299-007-0448-y

Tobimatsu, Y., and Schuetz, M. (2019). Lignin polymerization: how do plants manage the chemistry so well? Curr. Opin. Biotechnol. 56, 75-81. doi: 10.1016/j.copbio.2018.10.001

Vicente, O., and Boscaiu, M. (2018). Flavonoids: antioxidant compounds for plant defence and for a healthy human diet. Not. Bot. Horti Agrobot. ClujNapoca 46, 14-21. doi: 10.15835/nbha45210992

Vilasboa, J., da Costa, C. T., and Fett-Neto, A. G. (2018). Rooting of eucalypt cuttings as a problem-solving oriented model in plant biology. Prog. Biophys. Mol. Biol. 146, 85-97. doi: 10.1016/j.pbiomolbio.2018.12.007

Vilasboa, J., da Costa, C. T., Matsuura, H. N., and Fett-Neto, A. G. (2020). Rooting of cuttings of Passiflora suberosa, a medicinal passion fruit species: characterization and modulation by external biochemical factors. Isr. J. Plant Sci. 67, 40-51. doi: 10.1163/22238980-20191114

Wei, T., and Simko, V. (2017). R package "corrplot": Visualization of a Correlation Matrix (Version 0.84). Available at: https://github.com/taiyun/corrplot (Accessed October 10, 2020).

Weiner, J. (2020). pca3d: Three dimensional PCA plots. R package version 0.10.2. Available at: https://CRAN.R-project.org/package $=$ pca3d (Accessed October 10, 2020).

Xu, C., Shen, Y., He, F., Fu, X., Yu, H., Lu, W., et al. (2019). Auxin-mediated aux/IAA-ARF-HB signaling cascade regulates secondary xylem development in Populus. New Phytol. 222, 752-767. doi: 10.1111/nph.15658
Yoshimoto, K., Noutoshi, Y., Hayashi, K. I., Shirasu, K., Takahashi, T., and Motose, H. (2012). A chemical biology approach reveals an opposite action between thermospermine and auxin in xylem development in Arabidopsis thaliana. Plant Cell Physiol. 53, 635-645. doi: 10.1093/pcp/pcs017

Zhang, Y., Xiao, Z., Zhan, C., Liu, M., Xia, W., and Wang, N. (2019a). Comprehensive analysis of dynamic gene expression and investigation of the roles of hydrogen peroxide during adventitious rooting in poplar. BMC Plant Biol. 19:99. doi: 10.1186/s12870-019-1700-7

Zhang, G., Zhao, F., Chen, L., Pan, Y., Sun, L., Bao, N., et al. (2019b). Jasmonatemediated wound signalling promotes plant regeneration. Nat. Plants 5, 491-497. doi: 10.1038/s41477-019-0408-x

Zhishen, J., Mengcheng, T., and Jianming, W. (1999). The determination of flavonoid contents in mulberry and their scavenging effects on superoxide radicals. Food Chem. 64, 555-559. doi: 10.1016/S0308-8146(98)00102-2

Conflict of Interest: The authors declare that the research was conducted in the absence of any commercial or financial relationships that could be construed as a potential conflict of interest.

Copyright (c) 2021 Vilasboa, da Costa, Ransan, Mariath and Fett-Neto. This is an open-access article distributed under the terms of the Creative Commons Attribution License (CC BY). The use, distribution or reproduction in other forums is permitted, provided the original author(s) and the copyright owner(s) are credited and that the original publication in this journal is cited, in accordance with accepted academic practice. No use, distribution or reproduction is permitted which does not comply with these terms. 